\title{
Correction to: Dietary diversity score and cardio-metabolic risk factors: an updated systematic review and meta-analysis
}

\author{
Mostafa Qorbani ${ }^{1,2} \cdot$ Armita Mahdavi-Gorabi $^{2} \cdot$ Nasim Khatibi $^{3} \cdot$ Hanieh-Sadat Ejtahed $^{4,5} \cdot$ Maryam Khazdouz $^{6}$. \\ Shirin Djalalinia ${ }^{7,8} \cdot$ Amirhossein Sahebkar $^{9,10}$ - Mohammad Esmaeili-Abdar ${ }^{1}$. Motahareh Hasani ${ }^{6}$
}

Published online: 29 April 2021

(c) Springer Nature Switzerland AG 2021

\section{Correction to: \\ Eating and Weight Disorders - Studies on Anorexia, Bulimia and Obesity (2021) \\ https://doi.org/10.1007/s40519-020-01090-4}

Unfortunately, the second author name was incorrectly published in the original publication. The complete correct name is given below.

Armita Mahdavi-Gorabi

The original article has been corrected.
Publisher's Note Springer Nature remains neutral with regard to jurisdictional claims in published maps and institutional affiliations.

Mostafa Qorbani and Armita Mahdavi-Gorabi equally contributed as first authors.

The original article can be found online at https://doi.org/10.1007/ s40519-020-01090-4.

Motahareh Hasani

hasanimotahareh@yahoo.com

1 Non-Communicable Diseases Research Center, Alborz University of Medical Sciences, Karaj, Iran

2 Chronic Diseases Research Center, Endocrinology and Metabolism Population Sciences Institute, Tehran University of Medical Sciences, Tehran, Iran

3 Registered Dietitian, Shahid Sadoughi University of Medical Sciences, Yazd, Iran

4 Obesity and Eating Habits Research Center, Endocrinology and Metabolism Clinical Sciences Institute, Tehran University of Medical Sciences, Tehran, Iran

5 Endocrinology and Metabolism Research Center, Endocrinology and Metabolism Clinical Sciences Institute, Tehran University of Medical Sciences, Tehran, Iran
6 School of Public Health, Iran University of Medical Sciences, Tehran, Iran

7 Non-Communicable Diseases Research Center, Endocrinology and Metabolism Population Sciences Institute, Tehran University of Medical Sciences, Tehran, Iran

8 Deputy of Research and Technology, Ministry of Health and Medical Education, Tehran, Iran

9 Biotechnology Research Center, Pharmaceutical Technology Institute, Mashhad University of Medical Sciences, Mashhad, Iran

10 Neurogenic Inflammation Research Center, Mashhad University of Medical Sciences, Mashhad, Iran 